\title{
Consulta de enfermagem ao cliente hipertenso na estratégia saúde da família
}

\author{
Nursing consultation to hypertensive patients in family health strategy \\ Consulta de enfermería al cliente hipertenso en la estrategia salud de la familia
}

\author{
Antônia Sylca de Jesus Sousal; Marilia Braga Marques"I; Thereza Maria Magalhães Moreira"II; \\ Ana Danúsia Izidório Rodrigues de Araújo ${ }^{I V}$; Ana Zaira da Silvav; Ana Larissa Gomes MachadovI
}

\begin{abstract}
RESUMO: O estudo teve como objetivo descrever as ações realizadas pelos enfermeiros da atenção básica em Picos/Piauí voltadas ao acompanhamento do cliente com Hipertensão Arterial Sistêmica. Tratou-se de um estudo descritivo e de corte transversal, realizado com 26 enfermeiros atuantes na estratégia saúde da família, no período de agosto de 2010 a junho de 2011. Os dados foram coletados por meio de um questionário semiestruturado, construído com base em revisão de literatura, o qual foi validado com a utilização da técnica Delphi. Identificaram-se as características socioeconômicas e profissionais dos participantes, bem como as ações desenvolvidas na consulta de enfermagem ao hipertenso. Constatou-se deficiência na implementação do processo de enfermagem, assim como a necessidade de capacitação dos enfermeiros quanto à sua atuação na atenção primária, proporcionando um aperfeiçoamento da assistência a partir da reflexão sobre os aspectos contemplados na consulta de enfermagem à pessoa com hipertensão.

Palavras-Chave: Enfermagem; hipertensão; saúde da família; consulta.
\end{abstract}

ABSTRACT: This study aims at describing the actions of nurses from a primary care unit in Picos, Piauí, Brazil, regarding the care of patients suffering from Systemic Arterial Hypertension. This is a descriptive, cross-sectional study, done with 26 nurses who work with family health. The study was conducted from August, 2010 to June, 2011. Data were collected by means of a semi structured questionnaire on the basis of literature review, and were validated with the use of the Delphi technique. The socioeconomical and professional characteristics of the participants were assessed, as well as the actions performed by the nurses on patients with hypertension. We found that the implementation of the nursing processes is deficient, and the improvement of nursing techniques for acting in primary care is necessary. Assistance must be improved out of the observation of aspects of the treatment nurses give to patients with hypertension.

Keywords: Nursing; hypertension; family health; consultation.

RESUMEN: Este estudio tuvo como objetivo describir las acciones realizadas por los enfermeros de atención básica en Picos/ Piauí dirigidas al seguimiento del cliente con Hipertensión Arterial Sistémica. Se realizó un estudio descriptivo y de corte transversal, realizado con 26 enfermeros que actúan en la estrategia de salud de la familia, de agosto de 2010 a junio de 2011. Los datos se recabaron por medio de un cuestionario semiestructurado, construido a través de una revisión de la literatura, que fue validado mediante el empleo de la técnica Delphi. Se identificaron las características socioeconómicas y profesionales de los participantes, así como las acciones desarrolladas en la consulta de enfermería a los pacientes hipertensos. Se constató la deficiencia en la implementación del proceso de enfermería, así como la necesidad de formación de enfermeros en lo referente a su actuación en la atención primaria, produciendo un perfeccionamiento en la asistencia a partir de la reflexión sobre los aspectos contemplados en la consulta de enfermería a personas con hipertensión.

Palabras Clave: Enfermería; hipertensión; salud de la familia; consulta.

\section{INTRODUÇÃO}

A hipertensão arterial sistêmica (HAS) é uma síndrome multifatorial, na qual interações complexas entre fatores genéticos, ambientais e psicossociais causam elevação da pressão arterial, consequência da alta prevalência nacional e mundial é considerada como um dos principais fatores de risco cardíacos, cerebrovasculares e complicações renais ${ }^{1,2}$.

Entre os anos de 1990 e 2004, a prevalência da doença em algumas localidades no Brasil apresentou-se de $22,3 \%$ a $43,9 \%^{3}$, atingindo mais de $30 \%$ da população

IEnfermeira. Mestre em Enfermagem. Docente da Universidade Federal do Piauí. Floriano, Piauí, Brasil. e-mail: sylcasousa88@hotmail.com.

"Enfermeira. Doutoranda do Programa de Pós-graduação em Enfermagem da Universidade Federal do Ceará. Docente da Universidade Federal do Ceará. Fortaleza, Ceará, Brasil. e-mail: marilia@ufpi.edu.br.

IIIEnfermeira. Doutora em Enfermagem. Docente da Universidade Estadual do Ceará. Líder do Grupo de Pesquisa Epidemiologia, Cuidados em Cronicidades e Enfermagem. Fortaleza, Ceará, Brasil.e-mail: tmmmoreira@yahoo.com.

IVEnfermeira. Membro do Grupo de Pesquisa em Saúde Coletiva. Picos, Piauí, Brasil. e-mail: anadanusia 15@hotmail.com.

vEnfermeira. Mestranda do Programa de Pós-graduação em Cuidados Clínicos em Enfermagem e Saúde. Fortaleza, Ceará, Brasil. e-mail: anazaira18@hotmail.com.

VIEnfermeira. Doutoranda do Programa de Pós-graduação em Enfermagem da Universidade Federal do Ceará. Docente da Universidade Federal do Piauí. Picos, Piauí, Brasil. e-mail: analarissa2001@yahoo.com.br. 
adulta e mais de 50\% dos idosos no país ${ }^{4}$. Estratégias como mudanças no estilo de vida e nos hábitos alimentares são de fundamental importância para evitar os fatores de risco determinantes da hipertensão. Por isso, o diagnóstico precoce dessa patologia é de grande interesse para prevenir as suas complicações ${ }^{5}$.

Observa-se alto índice do controle da pressão arterial entre os usuários acompanhados na atenção primária quando comparado a outros modelos de atenção, mostrando impacto positivo da atuação dos profissionais nesse nível de assistência à saúde ${ }^{6}$.

Como integrante da equipe na Estratégia Saúde da Família (ESF), o enfermeiro deve desenvolver sua prática favorecendo a reorganização da assistência básica ao cliente hipertenso, contemplando o desenvolvimento de ações de prevenção, promoção e reabilitação da saúde do indivíduo. $\mathrm{O}$ atendimento por meio de consultas de enfermagem ajuda a atingir excelência no cuidado, pois as necessidades do indivíduo são avaliadas continuamente. A implementação de um programa estruturado proporciona, também, a possibilidade de avaliação crítica do cuidado, facilitando os ajustes clínicos e a comunicação multidisciplinar ${ }^{7,8}$.

$\mathrm{Na}$ ESF, a enfermeira se destaca como propagadora de informações e esclarecimentos acerca da importância dos hábitos saudáveis para o controle da pressão arterial e contribui na implementação de intervenções favoráveis à saúde. Ela integra grupos multiprofissionais com ações assistenciais e educativas conjuntas e implanta programas e consultas, aprofundando seu corpo de conhecimento?

Incumbe a este profissional a liderança na execução e avaliação do Processo de Enfermagem (PE), na qual a definição das intervenções ocorre a partir do estabelecimento de um diagnóstico de enfermagem (DE), de modo a classificar o cuidado prestado com uma linguagem padronizada para propiciar uma sistematização das informações e da assistência de enfermagem. Dessa forma, o planejamento e a implementação do cuidado de enfermagem são individualizados, auxiliando efetivamente na melhoria da situação de saúde. As intervenções podem ser realizadas em diferentes ambientes de cuidado, com metodologias e ferramentas variadas, aplicadas a pacientes e familiares de pacientes com diversos problemas de saúde ${ }^{8-11}$.

As consultas de enfermagem predominam sobre as consultas médicas na atenção primária aos usuários hipertensos ${ }^{12}$. No entanto, alguns aspectos não vêm sendo abordados durante as consultas, podendo acarretar prejuízos no controle da doença e na prevenção de complicações ${ }^{13}$. Faz-se necessário que os enfermeiros atuantes na ESF desenvolvam habilidades específicas para a realização satisfatória da consulta de enfermagem à pessoa com hipertensão.

Em vista do exposto, o objetivo do presente estudo foi descrever as ações realizadas pelos enfermeiros da atenção primária voltadas para o acompanhamento da pessoa com HAS.

\section{REVISÃo DE LITERATURA}

A HAS é uma condição clínica multifatorial caracterizada por níveis elevados e sustentados de pressão arterial (PA). Associam-se, frequentemente, alterações funcionais e/ou estruturais dos órgãos-alvo (coração, encéfalo, rins e vasos sanguíneos) e alterações metabólicas, com consequente aumento do risco de eventos cardiovasculares fatais e não fatais ${ }^{4}$.

Entre os fatores de risco para o desenvolvimento da HAS encontram-se a idade, sexo, raça, história familiar, ingestão alimentar rica em gorduras, acréscimo de sal aos alimentos, obesidade, sedentarismo, estresse, tabagismo, etilismo e uso de anticoncepcionais orais ${ }^{6}$. Estes fatores são os principais agravantes das patologias cardíacas e cerebrovasculares e complicações renais ${ }^{13}$.

O elevado percentual de comorbidades da HAS surge com o diagnóstico tardio e tratamento insuficiente, demandando a necessidade de investigação das práticas de enfermagem voltadas ao cuidado das pessoas com este agravo ${ }^{12}$.

Quando se discute a importância da abordagem multidisciplinar na atenção à pessoa com HAS, notase a necessidade de modificação das práticas em saúde, pois se trata de um agravo multifatorial que requer uma assistência pautada na interdisciplinaridade para maior efetividade do tratamento ${ }^{14}$.

É necessário o acompanhamento constante, por meio de consultas de rotina para que aspectos como vida, tratamento não farmacológico, seus efeitos adversos, hábitos de vida pessoais e familiares e o controle da pressão arterial, sejam constantemente checados pelo enfermeiro e reforçados a cada encontro, considerando que incorporar mudanças no estilo de vida, essenciais para que as demandas de autocuidado sejam satisfeitas, exige dedicação e motivação do indivíduo ${ }^{15-17}$.

As ações de saúde desenvolvidas para os indivíduos com HAS devem transcender as práticas convencionais adotadas no âmbito da atenção primária. Ao realizar atividades educativas que facilitem a promoção da saúde, estimulando-os ao autocuidado, deve-se explorar, além da consulta individual, a abordagem grupal. Os usuários com HAS não são consumidores apenas das orientações nos grupos, são agentes/ coprodutores de um processo de aprendizagem, possuindo uma dupla dimensão: são, ao mesmo tempo, objeto de trabalho dos agentes educativos e sujeitos de sua própria educação ${ }^{18}$.

O enfermeiro, na sua prática profissional, deve atender essa clientela, sistematizando suas ações, sendo necessária a implementação do PE, a fim de que seu trabalho e conhecimento conduzam ao repensar contínuo da prática profissional ${ }^{19}$, desenvolvendo ações que transcendem o modelo biomédico ainda muito enraizado na prática assistencial à pessoa com $\mathrm{HAS}^{20}$. 


\section{Metodologia}

Trata-se de um estudo descritivo ${ }^{21}$, realizado com enfermeiros atuantes nas Unidades da ESF do município de Picos-PI, no período de agosto de 2010 a junho de 2011.

A população compôs-se por 30 enfermeiros que atuavam na zona urbana e rural do município. Diante da dificuldade de acesso às unidades da zona rural, os sujeitos foram selecionados de forma aleatória. Participaram da pesquisa 26 enfermeiros das unidades da zona urbana e 6 enfermeiros atuantes na zona rural.

$\mathrm{O}$ estudo foi composto por quatro fases. $\mathrm{Na}$ primeira, realizou-se revisão de literatura para construção do instrumento de coleta de dados, um questionário que contemplou dados socioeconômicos, perfil e experiência profissional dos participantes, bem como as ações desenvolvidas na consulta de enfermagem ao hipertenso na ESF.

$\mathrm{Na}$ segunda fase, as perguntas do questionário relacionadas às ações desenvolvidas na consulta de enfermagem foram validadas por especialistas utilizando a técnica Delphi, que consiste no julgamento sobre os itens apresentados, durante os quais um grupo de juízes toma conhecimento do conteúdo e o avalia utilizando questionários ${ }^{22}$.

$\mathrm{Na}$ terceira fase do estudo, foi realizado contato com os enfermeiros para coletar os dados acerca dos aspectos socioeconômicos e profissionais e agendar o acompanhamento de três consultas de enfermagem com pessoas hipertensas a serem observadas pelo pesquisador. Para guiar a observação, utilizou-se o questionário validado pelos juízes acerca das ações a serem contempladas na consulta de enfermagem a pessoa com HAS.

Na quarta fase, os enfermeiros foram convidados a participar de uma entrevista realizada na unidade de saúde em momento oportuno para o participante indagando-o: quais os aspectos contemplados na consulta de enfermagem ao hipertenso? A fim de garantir o anonimato dos participantes do estudo, os mesmos foram identificados por ordem de realização das consultas, por meio do uso de termos (E1, E2, E3, ..., E26).

Para análise dos dados utilizou-se estatística descritiva. A apresentação deu-se a partir de figuras e tabelas. A pesquisa teve prosseguimento após cada usuário ter conhecimento acerca da metodologia e dos objetivos do estudo, afirmando anuência por meio da assinatura do Termo de Consentimento Livre e Esclarecido (TCLE). O desenvolvimento da pesquisa seguiu os princípios expressos na Resolução no ${ }^{\circ}$ 466/12 do Conselho Nacional de Saúde (CNS), que estabelece os preceitos éticos das pesquisas envolvendo seres humanos ${ }^{23}$, e fez parte de projeto intitulado Práticas de Enfermagem na Prevenção e Controle de Doenças Crônicas Não-transmissíveis, $\mathrm{n}^{\circ}$ CAAE: 0269.0.045.000.10.

\section{Resultados e Discussão}

Os dados referentes ao perfil socioeconômico e profissional dos enfermeiros participantes da pesquisa são apresentados na Tabela 1.

TABELA 1: Caracterização socioeconômica e profissional dos enfermeiros, Picos-PI, mar-abril, 2011. ( $N=26)$

\begin{tabular}{|c|c|c|}
\hline $\begin{array}{c}\text { Caracterização } \\
\text { socioeconômica e } \\
\text { profissional }\end{array}$ & $f$ & $\%$ \\
\hline \multicolumn{3}{|l|}{ Sexo } \\
\hline Masculino & 1 & 3,8 \\
\hline Feminino & 25 & 96,2 \\
\hline \multicolumn{3}{|l|}{ Idade (anos) } \\
\hline 25 а 30 & 13 & 50,0 \\
\hline$>31$ a 50 & 10 & 38,5 \\
\hline$>50$ & 3 & 11,5 \\
\hline \multicolumn{3}{|l|}{ Estado civil } \\
\hline Solteiro & 12 & 46,2 \\
\hline Casado & 14 & 53,8 \\
\hline \multicolumn{3}{|c|}{ Renda em salários mínimos } \\
\hline 1 a 5 & 13 & 50,0 \\
\hline$>5$ & 13 & 50,0 \\
\hline \multicolumn{3}{|c|}{ Desenvolve outra atividade } \\
\hline Sim & 12 & 46,2 \\
\hline Não & 14 & 53,8 \\
\hline \multicolumn{3}{|l|}{ Exercício atual na ESF } \\
\hline Concurso público & 19 & 73,1 \\
\hline Serviço prestado & 7 & 26,9 \\
\hline \multicolumn{3}{|l|}{ Curso de imersão na ESF } \\
\hline Sim & 6 & 23,1 \\
\hline Não & 20 & 76,9 \\
\hline
\end{tabular}

*Salário Mínimo= R\$545,00

Houve predominância do sexo feminino entre os enfermeiros investigados, 25 (96,2\%), com idade compreendida entre 20 e 30 anos, 13 (50,0\%), casadas, 14 (53,8\%), demonstrando profissionais jovens atuando na ESF do município. Percebeu-se também que $12(46,2 \%)$ enfermeiros desenvolviam outro tipo de trabalho, evidenciando que a atuação na atenção primária não é exclusiva em sua vida profissional. Sabe-se que para atuar como membro integrante da equipe da ESF, o enfermeiro deve trabalhar cerca de oito horas diárias ${ }^{24}$, mas não se requer exclusividade nesta atividade.

Observou-se predominância da atuação dos profissionais por meio de concurso público, 19 $(73,1 \%)$, embora apresente um número pouco expressivo, 7 (26,9\%), daqueles que exercem a profissão por serviço prestado. $\mathrm{O}$ trabalho na ESF pressupõe que o profissional resida no município de atuação, sendo essa condição favorecida pelo processo de ingresso por meio do concurso público ${ }^{24}$. 
Entre os enfermeiros entrevistados, 20 (76,9\%) não realizaram curso de imersão prévia à contratação para atuação com o público atendido por meio dos programas disponibilizados nas USF. Preconiza-se que a capacitação deve se iniciar concomitantemente ao início do trabalho das ESF por meio do Curso Introdutório para toda a equipe ${ }^{24}$.

Durante a observação das consultas de enfermagem ao cliente com HAS, pode-se constatar que $14(53,4 \%)$ enfermeiros realizavam consulta de enfermagem ao cliente com hipertensão sistematicamente, mas se observou ainda um número bastante elevado de profissionais, 12 $(46,1 \%)$, que não realizavam a consulta.

As falas dos enfermeiros referentes às etapas do processo de enfermagem contempladas no atendimento a pessoa com HAS obtidas a partir das entrevistas estão sumarizadas na Figura 1.

Os aspectos que os enfermeiros referiram realizar na consulta de enfermagem foram: a primeira etapa do processo, ou seja, o histórico de enfermagem, colhendo dados e oferecendo informações a respeito das medidas antropométricas, os hábitos (tabagismo/

\begin{tabular}{|c|c|c|}
\hline $\begin{array}{l}\text { Etapas do processo } \\
\text { de enfermagem }\end{array}$ & Falas dos enfermeiros & \\
\hline \multirow[t]{3}{*}{ Histórico } & $\begin{array}{l}\text { Depois da abordagem inicial a gente faz a questão do } \\
\text { exame físico... onde se faz a aferição dos sinais vitais, } \\
\text { circunferência abdominal, peso [...] (E5) }\end{array}$ & $\begin{array}{c}\text { Medidas } \\
\text { antropométricas }\end{array}$ \\
\hline & $\begin{array}{l}\text { A questão do fumo e do alcoolismo eles às vezes até } \\
\text { negam na consulta, mas a gente sabe que ainda é um } \\
\text { predisponente muito forte para vir a ser hipertenso (E2) }\end{array}$ & $\begin{array}{c}\text { Hábitos } \\
\text { (tabagismo/etilismo) }\end{array}$ \\
\hline & $\begin{array}{l}\text { Na anamnese a gente verifica os hábitos dos pacientes, } \\
\text { seus antecedentes [...] (E6) }\end{array}$ & $\begin{array}{c}\text { Hábitos/ } \\
\text { Antecedentes pessoais }\end{array}$ \\
\hline $\begin{array}{l}\text { Diagnóstico de } \\
\text { enfermagem }\end{array}$ & Não houve falas referentes a esta etapa. & \\
\hline Planejamento & Não houve falas referentes a esta etapa. & \\
\hline \multirow[t]{6}{*}{ Implementação } & $\begin{array}{l}\text { No cliente hipertenso tem que ser abordado vários } \\
\text { aspectos. Começando com a dieta, com a alimentação, a } \\
\text { gente tem que tá orientando em relação ao sal, à } \\
\text { gordura, à fritura, a refrigerantes, a doces, ao açúcar [...] } \\
\text { (E3) }\end{array}$ & Alimentação \\
\hline & $\begin{array}{l}\text { Outro ponto é a prática de atividade física que ajuda no } \\
\text { controle dos níveis pressóricos, e eu recomendo que } \\
\text { meus pacientes realizem caminhadas pelo menos três } \\
\text { vezes por semana. (E4) }\end{array}$ & Atividade física \\
\hline & $\begin{array}{l}\text { [...] enfatizar realmente como a fisiopatologia da doença } \\
\text { enfatiza a adesão a terapia farmacológica [...] (E1) }\end{array}$ & Terapia farmacológica \\
\hline & $\begin{array}{l}\text { De modo geral, na consulta de Enfermagem ao } \\
\text { hipertenso entra a questão... da orientação para } \\
\text { realização de exames }[. . .](E 7)\end{array}$ & Realização de exames \\
\hline & $\begin{array}{l}\text { [...] eles precisam entender o que aquela pressão } \\
\text { descompensada, aquela pressão alta vai acarretar com o } \\
\text { tempo [...] (E8) }\end{array}$ & Complicações \\
\hline & $\begin{array}{l}\text { Em relação ao programa HIPERDIA, realizamos palestras } \\
\text { educativa uma vez ao mês [...] (E9) }\end{array}$ & Educação em saúde \\
\hline Evolução & $\begin{array}{l}\text { [...] eu creio que seja muito importante também o } \\
\text { acompanhamento na unidade. (E10) }\end{array}$ & Seguimento \\
\hline
\end{tabular}

FIGURA 1: Falas dos enfermeiros sobre a consulta ao hipertenso, conforme as etapas do processo de enfermagem, Picos, mar-abril, 2011. 
etilismo) e os antecedentes pessoais. Realizavam também a implementação, por meio de orientação sobre hábitos alimentares, atividades físicas, as terapias farmacológicas, a realização de exames, complicações sobre a patologia e atividades de educação em saúde; por último, a evolução, acompanhando o paciente de forma rotineira na Unidade de Saúde.

As ações relatadas pelos enfermeiros levam à reflexão acerca da condução da consulta de enfermagem que não deve se restringir apenas a estas etapas, nas quais está sendo enfocada apenas a doença, devendose atender a pessoa de forma integral.

Em estudo de análise de processos de enfermagem no Brasil ${ }^{25}$, percebe-se que o processo de enfermagem (PE) perde sua essência ao ser abordado de forma fragmentada, o que se deu na maioria dos estudos, 74 (66\%). Estes não abordaram as etapas integradas deste processo, mas apenas algumas etapas separadas, independentes e fragmentadas. Dentre estes fragmentos do PE, a maioria, $16(14,3 \%)$, referiu-se ao diagnóstico de enfermagem, denotando a preocupação dos profissionais em trabalhar com respostas humanas a processos e quadros patológicos, numa visão ainda reducionista do ser humano.

A partir da observação das consultas de enfermagem, durante a coleta de dados, percebeu-se quais aspectos foram avaliados pelos enfermeiros, apresentados na Tabela 2.

TABELA 2: O fazer dos enfermeiros na consulta de enfermagem ao hipertenso na ESF. Picos-PI, mar-abril, 2011. $(\mathrm{N}=42)^{(*)}$

\begin{tabular}{lrr}
\hline Consulta ao hipertenso na ESF & $\mathrm{f}$ & $\%$ \\
\hline Anamnese & 13 & 30,9 \\
Hábito de fumar e beber & 26 & 61,9 \\
Ingestão excessiva de sal & 12 & 28,6 \\
Antecedentes pessoais & 23 & 54,8 \\
Sedentarismo e aumento de peso & 2 & 4,8 \\
Uso de medicamentos hipertensivos & 4 & 9,6 \\
$\quad$ Exames laboratoriais anteriores & & \\
Exame físico & 42 & 100 \\
Pressão arterial & 33 & 78,6 \\
Peso e altura & 3 & 7,1 \\
Peso & 16 & 39,0 \\
Cálculo do IMC & 3 & 7,1 \\
Abdômen & 3 & 7,1 \\
Pulsos carotídeos & 3 & 7,1 \\
Fáceis e pescoço & 3 & 7,1 \\
Precórdio & 1 & 2,4 \\
Circunferência abdominal & 1 & 2,4 \\
Pés e pernas & & \\
Métodos de aferição PA & 40 & 95,2 \\
Auscultatório & 2 & 4,8 \\
Auscultatório e palpatório & & \\
Posição de verificação PA & 42 & 100,0 \\
Sentado & & \\
Orientações na consulta & 33 & 78,6 \\
Padrão nutricional & 28 & 66,7 \\
Atividade física & 12 & 28,6 \\
Abandono do tabagismo & 34 & 81,0 \\
Uso correto da medicação & & \\
\hline
\end{tabular}

${ }^{(*)}$ Foram observadas duas consultas por enfermeiro.
Observa-se que, na anamnese, a investigação da ingestão excessiva do sal e o sedentarismo/aumento de peso foram priorizados, respectivamente caracterizados por $26(61,9 \%)$ e 23 (54,8\%) ocorrências, e que os demais itens fundamentais nesta fase da consulta foram pouco enfatizados.

A população brasileira apresenta um padrão alimentar rico em sal, açúcar e gorduras, sendo que alguns fatores - como o excesso de peso - têm sido correlacionados com a elevação da pressão arterial desde idades jovens. Tal quadro exige maior investigação do profissional durante a consulta a fim de contribuir para a detecção e tratamento precoces do agravo ${ }^{4}$.

Em relação aos aspectos contemplados durante o exame físico, os enfermeiros, em sua totalidade, realizaram a aferição da pressão arterial com o paciente na posição sentada, mas $40(95,2 \%)$ profissionais somente utilizaram o método auscultatório, o que diverge com o priorizado na literatura ${ }^{4}$, devendo-se contemplar os métodos auscultatório e palpatório. Em pesquisa congênere ${ }^{15}$, destacou-se no exame físico o fato das técnicas como a palpação e ausculta estarem sendo negligenciadas pelos enfermeiros.

Dentre os padrões que contemplam a consulta ao cliente hipertenso, a atividade mais realizada pelos enfermeiros neste estudo foram as orientações sobre o uso correto da medicação, 34 (81,0\%), a manutenção do padrão nutricional, $33(78,6 \%)$, a realização regular de atividades físicas, 28 (66,7\%) e o estímulo ao abandono do tabagismo, 12 (28,6\%).

Em outro estudo relacionado à consulta de enfermagem com pessoas com $\mathrm{HAS}^{14}$, encontraram-se 22 registros referentes à ação orientar, representando apenas 2,1\% do total de registros de intervenções da pesquisa. Consideram-se ainda incipientes as intervenções relacionadas à conversa e sensibilização do cliente com HAS na adoção de comportamentos favoráveis ao controle de fatores de risco, fato que pode ser explicado pela complexidade envolvida com o ato de lidar com questões comportamentais e subjetivas relacionadas ao tratamento.

\section{Conclusão}

O estudo destaca lacunas na implementação do processo de enfermagem durante a realização de consultas de enfermagem ao hipertenso na atenção primária. Essa constatação evidencia a necessidade de educação continuada dos enfermeiros na atenção básica para o cuidado sistematizado à pessoa com hipertensão.

Constata-se, ainda, que a realização da consulta de enfermagem ao hipertenso não se tornou rotina nos serviços de atenção primária investigados. Ressalta-se, contudo, que alguns enfermeiros realizam a consulta e sensibilizam os clientes sobre sua condição de saúde, pactuando com eles metas e planos de como dar seguimento ao tratamento. 
Esta investigação apresenta como limitação a reduzida amostra analisada, dada a dificuldade de acesso aos profissionais e a algumas unidades básicas. Sugere-se, portanto, a realização de pesquisas de intervenção que colaborem com a mudança das práticas realizadas neste cenário pelo enfermeiro a partir de cursos e treinamentos realizados em serviço.

\section{REFERÊNCIAS}

1.Berardinelli LMM, Figueiredo TFL, Oliveira AS, Santos I, Giron MN, Ramos JP. Hipertensão arterial e conhecimento popular: potencializando o cuidado. Rev enferm UERJ. 2013; 21: 446-51.

2.Fava SMCL, Figueiredo AS, Franceli AB, Nogueira MS, Cavalari E. Diagnóstico de enfermagem e proposta de intervenções para clientes com hipertensão arterial. Rev enferm UERJ. 2010; 18: 536-40.

3.Ministério da Saúde (Br). Departamento de Ações Programáticas Estratégicas. Plano de Reorganização da Atenção à Hipertensão Arterial e ao Diabetes mellitus. Brasília (DF): Editora MS; 2001.

4.Sociedade Brasileira de Cardiologia, Sociedade Brasileira de Hipertensão, Sociedade Brasileira de Nefrologia. VI Diretrizes Brasileiras de Hipertensão. Arq Bras Cardiol. 2010; 95: 1-51.

5.Almeida APR, Silva FAA, Santiago JCS, Moreira TMM, Oliveira ASS, Machado ALG. Caracterização sociodemográfica e hábitos de vida de acadêmicos: identificando fatores de risco para hipertensão arterial. Rev enferm UERJ. 2013; 21: 760-5.

6.Gomes TJO, Silva MVR, Santos AA. Controle da pressão arterial em pacientes atendidos pelo programa hiperdia em uma unidade de saúde da família. Rev Bras Hipertens. 2010; 17: 132-9.

7.Curcio R, Lima MHM, Torres HC. Protocolo para consulta de enfermagem: assistência a pacientes com diabetes melittus tipo 2 em insulinoterapia. Rev Gaúcha Enferm. 2009; 30: 552-7.

8.Vieira RQ, Oliveira EC, Lima JV, Rubbo AB. História da assistência de enfermagem brasileira acerca da hipertensão arterial (1949-1988). Hist Enf Rev Eletr (HERE). 2014; 5: 67-82.

9.Santos JC, Florêncio RS, Oliveira CJ, Moreira TMM. Adesão do idoso ao tratamento para hipertensão arterial e intervenções de enfermagem. Rev RENE, 2012; 13: 343-53.

10.Scain SF, Franzen E, Santos LB, Heldt E. Acurácia das intervenções de enfermagem para pacientes com diabetes mellitus tipo 2 em consulta ambulatorial. Rev Gaúcha Enferm. 2013; 34:14-20.
11.Barreto MS, Marcon SS. Participação o familiar no tratamento da hipertensão arterial na perspectiva do doente. Texto contexto enferm. 2014; 23: 38-46.

12.Hoepfner C, Franco SC. Inércia clínica e controle da hipertensão arterial nas unidades de atenção primária à saúde. Arq Bras Cardiol. 2010; 95: 223-9.

13.Barros ALBL, Vieira FS, Assis CC, Zeitoun SS. Alterações do nível pressórico e fatores de risco em graduandos de enfermagem. Acta Paul Enferm. 2009; 22:773-8.

14.Costa FBC, Oliveira CJ, Araújo TL. Intervenções de enfermagem em portadores de hipertensão arterial: análise documental. Rev enferm UERJ. 2008; 16:482-8. 15.Manzini FC, Simonetti JP. Consulta de enfermagem aplicada a clientes portadores de hipertensão arterial: uso da teoria do autocuidado de Orem. Rev Latino-Am Enfermagem. 2009;17:114-20.

16.Alves EBL. Acompanhamento de enfermagem aos idosos hipertensos: relato de experiência. InterScientia. 2014; 2: 3-15.

17. Borges JWP, Pinheiro NMG, Souza ACC. Hipertensão comunicada e hipertensão compreendida: saberes e práticas de enfermagem em um Programa de Saúde da Família de Fortaleza, Ceará. Ciência \& Saúde Coletiva. 2012; 17: 179-89.

18.Toledo MM, Rodrigues SC, Chiesa AM. Educação em saúde no enfrentamento da hipertensão arterial: uma nova ótica para um velho problema. Texto contexto enferm. 2007; 16: 233-8.

19.Felipe GF, Abreu RNDC, Moreira TMM. Aspectos contemplados na consulta de enfermagem ao paciente com hipertensão atendido no programa saúde da família. Rev esc enferm USP. 2008; 42:520-7.

20.Reiners AAO, Nogueira MS. Conscientização do usuário hipertenso para a adesão ao tratamento. Rev Latino-Am Enfermagem. 2009; 17: 59-65.

21.Andrade MM. Pesquisa científica: noções introdutórias. In: Andrade MM Introdução à metodologia do trabalho científico. $7^{\text {a }}$ ed. São Paulo: Atlas; 2006.

22.Duffield C. The delphi technique: a comparison of results obtained using two expert panels. Lut J Nurs Stud, 1993; 30: 277-37.

23. Conselho Nacional de Saúde (Br). Resolução n⿳⺈ 466/12. Dispõe sobre diretrizes e normas regulamentadoras de pesquisas envolvendo seres humanos. Brasília (DF): Conselho Nacional de Saúde; 2012.

24.Ministério da Saúde (Br). Estratégias para o cuidado da pessoa com doença crônica: hipertensão arterial sistêmica. Brasília (DF): Editora MS; 2013.

25.Aguiar DT, Meneses GGM, Pinto FJM, Moreira TMM, Fialho AVM. Processo de enfermagem: análise de dissertações e teses de enfermagem no Brasil de 1979. 2007. Cogitare Enferm. 2010; 15: 742-8. 